\title{
The Application of Chinese Classical Garden in Interior Design
}

\author{
Hongxing $\mathrm{Yi}^{1, \mathrm{a}^{*}}$ and Wenjun Wang ${ }^{2, \mathrm{~b}}$ \\ ${ }^{1}$ City College of WUST, Wuhan, China \\ alucyyhxc@126.com, b15827425957@163.com
}

*The Corresponding author

\begin{abstract}
Keywords: Garden; Landscape; Interior design; Chinese style; Classical
\end{abstract}
\begin{abstract}
The landscape is composed of human and art processing. Chinese classical garden is a landscape design with oriental characteristics. China classical garden beauty is rooted in nature and transcending nature. It is a unification of the natural landscape and cultural landscape. With the continuous improvement of the concept of interior design, a large number of landscape designs have been introduced into the interior design, forming a new type of indoor landscape. The beauty of Chinese classical gardens is diverse. In the design, not only to combine the Chinese style of interior design beauty rules, but also pay attention to follow the laws of Chinese classical garden beauty.
\end{abstract}

\section{The Aesthetic Thought of Chinese Classical Gardens}

Natural Beauty. Chinese classical garden has a rich connotation and unique artistic conception. It is in pursuit of natural spiritual realm as the ultimate goal, so as to achieve "by man, heaven" aesthetic taste. It took China cultural connotation, is a vivid portrayal of a nation's inner spirit. It emphasizes "imitate the nature". It is against the law and against nature of artificial polish. It embodies the artificial natural beauty as a garden theme. Chinese aesthetics of classical garden is the overall pursuit of natural beauty, the pursuit of local natural beauty.

Since ancient times, the human society has been outing, climbing, spring outing, flowers and other practices, and has continued to this day. People love of plants and flowers are often filled in poetry. Su Dongpo once said: "would rather eat no meat, can not live without bamboo"; Du Fu's poem: "living will Linquan, build house edge water". Obviously, people have unlimited pursuit of natural beauty. Advocating nature, enjoy nature and nature respiration is an important part of people's life.

Artistic Beauty. The connotation of "Chinese classical garden artistic conception" is the core of Chinese garden of traditional style and features. What is the meaning of "artistic conception"? It is love. The environment is the object. It is from the scene. The scene is made of heart. Scene blend and produce mood. The ancients said: "love and circumstances, the situation becomes more and more deep, scenery and feeling, is often the new king." So "artistic conception" to the art to the soul, filled with anger. It is for the scene of emotion, attitude to the screen. The image has become subtle. The landscape looked deep. It has floated on objects outside of love, mysterious and voice, the environment, the special charm of taste. In aesthetic activities, highly played a dynamic role of imagery thinking, stimulate the mind create much rich artistic taste than the actual scenery. In experiencing feelings, let the tourists from the heart into the feeling into the body to empathy, hint of fascinating, thought-provoking, to deep the lofty spirit of the world. This is the aesthetic psychological structure of traditional landscape art Chinese classical gardens. They are built in the inner realm on the basis of "heart induction" and "Xing to God" aesthetic value.

Some schools even into the practice of Zen Wu one stone, every tree and bush, the Buddhist pursuit of realm of understanding. Create the inner world in a small courtyard, the so-called "one world, one a bodhi tree." The abstract mean has achieved a strong sense of more than five direct and natural compatible with the tacit understanding, and lead people to the mysterious mystery introspection realm. Chinese classical garden is rich. Due to the mountains towering like Taishan drone momentum, make water to rivers and lakes like good soup charm. This is in order to show the close to nature, nature counterattack of the hermit life environment, but also to their traditional "mountains and 
water" concept. It is the imitation of nature, but not too artificial. In this garden, can achieve the "physical and mental dust away, sitting in the left time" is the pursuit of "lyre to see the cranes, the pillow surface to return" and "wild life sitting moss student seats, high sleep hanging bamboo clothes" interesting. Chinese classical landscape stone love, water, flowers and trees also have a touch of charm. The road is the size of corner trees, broken branches. Several strains of mountain trees, tall bamboo a cluster, are simulated. Chinese wood bamboo and stone classical gardens in deep twists and turns, they are wonderful in subtle and hidden so, "how deep is the courtyard" and "remote willow is outside, like every lotus no way to pass" and other famous quotes.

China classical gardens pursuit in philosophy is a kind of chaotic no image, pure, harmony and balance of yin and Yang, and nature between maintaining the harmonious, harmonious interdependence. All kinds of natural objects in the form of objective attributes, such as line, shape, proportion, combination, does not occupy the main status in the aesthetic consciousness, but mainly to the subjective grasp of the natural space. On the way, with the implicit inexhaustible, the path winds along mountain ridges, hidden realm. It is a kind of imitative nature, closed garden pursuing nature, and an "independence of paradise".

\section{Chinese Classical Garden Landscape Gardening Techniques}

According to the basic aesthetic thoughts reflect the natural beauty of the landscape theme and create artistic conception of the ancient gardening artists in the garden of the mountain, water, buildings, plants as material, from space to time of organic and natural combination, a garden with a flexible layout characteristics of random nature, in the long-term practice of landscape gardening in the following form basic principles:

A, meaning to connotative, evocative; two, hill pools to art beats nature, wan since opening day; three, landscape architecture should be coordinated with other elements and the surrounding landscape; four, landscape layout should have a sense of hierarchy, to prioritize.

The beauty of China classical landscape is based on introspection, subtle, refined, charming, quiet and indifferent, through the moment, focusing on feelings and spiritual understanding.

By China history, culture, philosophy, and the influence of the traditional aesthetics, China classical landscape forms, such as Chinese classical gardens as representative of the natural landscape, the landscape features of asymmetric equilibrium to curve as the natural landscape garden guide, a unique style known in the world.

\section{Artistic Combination of Chinese Classical Garden Landscape}

The relative independence and aesthetic characteristics of the various parts of the garden are presented through the ingenious and changeable artistic combination of landscape.

Blocking View. The garden scenery in the proper shelter, not to hide, flat, straight to the point, but if you want to see the big picture, must through the labyrinth circuitous, finally in the garden at the entrance of "dense willow trees and bright flowers." by King curb practices.

On the Scene. The so-called "scene" is one of the relatively. It is to each other to watch the scenery technique. The use of each set off against the scene depending on the situation, sometimes a spot in only one place on the scene, sometimes surrounded by around.

Point of View. According to the landscape properties, uses, combined with environmental characteristics were highly summarized, with a view to point out the word means scenery features. Landscape is the most common Chinese combination of classical gardens, often the garden mood deepened, and poetic enrichment plays a particularly important role. It can not only connotation the rich landscape, in addition to the landscape of the theme, people back to connect in the mind. In the form of a stone, stone plaque, antithetical couplet, and so on.

Hidden Scene. Hide the scene is a distant mood gardening practices. It is actually formed in the garden of the relatively closed small space, resulting in a spatial effect in the garden. The garden 
mountain the Summer Palace in Beijing after the Xiequ Yuan is a big garden in the garden. It is achieve aesthetic effect in the downtown. Beihai's Jingxinzhai is the use of hidden means of view, create an exquisite small Jiangnan garden, formed the garden park, hidden but beautiful spot of the art realm.

Borrowed Scenery. It is the scenery outside the park into the park, to enrich the content and artistic expression of ornamental garden. Borrowed Scenery has look by far, close look, look up, look down, borrowed and should be grading. The Summer Palace in Beijing borrow scenery in Xishan Mountain, Yuquan Mountain, called by far; the Humble Administrator's Garden in Suzhou by the garden outside the North Temple Pagoda, called borrowed by far; throughout the year, when sunny landscape garden is sometimes to borrow and borrow; and neighbor borrowed, by far just because of the garden and outside scene space distance varies.

Enframed Scenery. The garden building door, window, or canopy gaps hold together, like a variety of frames will be distant landscape or the humanities landscape box into which the picture produced wonderful effect, namely frame view. Shape box scene with other window openings, the different and varied one, or square or, circular, or flower shape, or objects shape, different style is to heighten the role for the expression of the conception of the box scene.

Imported Scenery. That is to attract visitors to visit the scene in the garden pavilion, mountain Pavilion, corridor, etc. can play the lead role. Xiling Seal-Engravers'Society in Hangzhou has a row of the press on the wall, outside the park through the park and the park scenery visible, a tour of interest.

Add Scenery. Between the garden scenery is too big or monotonous, will increase the number of views, to complement it, namely to add the Scenery. Add the scenery can enrich the landscape level; enhance the appeal of the depth of field. Add the scenery theme is rich, can be a stone peak, can also be flowers, trees, also can be a corner of the building.

In the Middle of the Scene. When the scenic spots in the distance, or natural mountain, or cultural buildings (such as tower bridge, etc.), itself is very ornamental value, but because there is no shelter and open sight is too monotonous, so often with rocks in the tour routes on both sides and both sides, trees or buildings as tourist attractions with compose, and the formation of guide line, the landscape around the background to gradually enter the visitors' view, thus increasing the search means, this scenery technique is the scene such as boating in the Summer Palace. With the mountain to the Suzhou River, the distance of the Suzhou bridge king, the rolling hills and facing each other from both sides of the beautiful forest beautiful scenery.

\section{The Application of Chinese Classical Garden Landscape in Modern Interior Design}

Gardening techniques in Chinese classical garden has creation of artistic conception, spatial layout and composition of elements. The landscape design skills including the theme of isolation and not plug, zigzag, scale happy ending without happy beginning, properly, by far the neighbor borrowed. These expressions are of great significance in the application of modern interior design.

The design idea, whether it is in the China classical garden art, or in the interior landscape art, "artistic conception" is one of the most intriguing propositions. Spatial implication of Chinese classical garden design, landscape design is the highest level. It reflects the day, three people's harmony and design aesthetics the natural ecological thought. The essence of Chinese is advocating classical garden artistic conception, and wonderful in connotation.

"Quiet, deep in the Buddhist temple garden" the beauty of artistic conception of the garden. In the Chinese concept of traditional culture, elegant environment with the regulation of life, saying the sexual function. The beauty of the environment is nothing more than the beauty of natural scenery. Nature has gathered all the good things, like the moon and stars, mountains and rivers, the wonderful form in different poses and with different expressions, with countless changes a quality suggestive of poetry or painting.

The "borrowed scenery" and " on the scene" is often used China in classical landscape gardening practices, to convey the mood change. Obscure, implicit borrowed scenery is the most widely used 
technique Chinese classical gardens. It is the garden outside or near or far scenery ingenious "borrowed" to the garden, become a part of the landscape. "The window containing the west mountain snow and door's breaking many ships", this is the ancient poet "by far".

Scene is in the scene, dynamic and static, large and small, curved and straight, and correspond to each other, enrich the connotation of landscape. In the layout, often Chinese garden the use of both inside and outside, we are taking Chinese practices in classical gardens, people involved in making natural movement eventually formed a complete process, such as garden stone technique, stone is static, rigid, natural materials without life, while the classical Chinese is thinking. It is intended to become a bridge between man and nature.

Different from the outdoor landscape, the indoor natural landscape can be used in addition to the outdoor landscape, but also can be used in the indoor landscape. At the same time, the indoor natural landscape itself is borrowed to become part of the interior landscape. The Song Dynasty painter Guoxi said in the "Lin Quan Gao Zhi": "the mountains walker, is expected to have, a tour, a home", "practical, hopefully, can swim, can be in, can let people return to nature". This is also the basic idea of the classical garden design

In China classical garden design, exquisite king, hidden, emphasizes the spirit and noble character, these ideas are often referred to in the interior landscape design nowadays, such as our common view windows, carved screen, with plants to symbolize noble character. Now this idea is further developed into our interior design. This is called "the construction of scenario, indoor space must have the situation of beauty, infection can deep heart. For example, the room space separated by the appropriate block, planted flowers, the curtain cloth plum, orchid, bamboo, chrysanthemum and other symbolic patterns. These Chinese elements with different colors, expresses the Chinese "artistic beauty" of nature and harmony.

The modern interior design, along with the rise of natural design, interior decoration is more and more widely used in the landscape of interior decoration engineering. Especially in high-rise buildings and indoor air conditioning in buildings, the natural scenery suitable from the outside into the room, the indoor garden given certain atmosphere, enrich and beautify the indoor the indoor space, active atmosphere, so as to enhance people's natural comfort and harmony of nature. Modern interior decoration garden has water bureau scenery, mountain stone scenery, ornamental plant landscape, landscape and garden pavilions, terraces and open halls, building and so on. In the design, application of landscape has made great progress. The natural landscape and artificial landscape were combined in the room, the formation of a certain scene. The interior space in the business club hotel, but in some private houses also began to be used.

\section{Summary}

Chinese classical garden famous overseas, has a long history, rich cultural content, distinctive personality, and colorful, highly artistic charm, and is the precious heritage of all mankind. It shows the Chinese people's wisdom and excellent aesthetic consciousness, is a bright pearl in the traditional culture. Chinese should be fully integrated into the China classical garden design in the modern interior design, the introduction of natural indoor, to combine the modern technology and ancient art better, design a space environment more fresh and elegant.

\section{Acknowledgement}

The search topic of City College of WUST 2016, landscape design-landscaping curriculum construction research and practice. Item number: 2016CYYBJY024

\section{References}

[1] J.H.Liu: Writer, (2014) No.11, pp.225-226 
[2] Y.Y.Zhang: Research on the application of Suzhou landscape art in interior design, (Southwest Jiaotong University Press, China and 2013)

[3] X.Zhu and Y.Chen: Modern Decoration (Theory), (2016) No. 1, pp.68-69

[4] H.Zhao and D.L.Zhao: Social Sciences Journal of Universities in Shanxi, (2011) No. 5, pp. 108-110

[5] J.Xie and N.Liu: Master, (2010) No.2, pp.198-198

[6] .Wang: Art of Design, (2003) No.1, pp.23-23

[7] Y.P.Wan: Science \& Technology Information, (2010) No.16, pp.271-272

[8] .Ma: Art Science and Technology, (2016) No.29, pp.304-304

[9] .T.Du: Study on the application of Chinese classical gardens in modern interior design, (Dalian Polytechnic University Press, China and 2013)

[10] .Li: Urban Construction Theory Research, (2014) No. 33, (In Chinese)

[11].H.Ta: Da Guan, (2015) No. 9, (In Chinese)

[12].X.Zhen: Modern Decoration (Theory), (2012) No.1, pp.40-41, (In Chinese)

[13].Zhao: Journal of Technology, (2013) No.7, pp.57-57, (In Chinese) 